\title{
Mixing old and young: enhancing rejuvenation and accelerating aging
}

\author{
Ashley Lau, ${ }^{1}$ Brian K. Kennedy, ${ }^{2,3,4,5}$ James L. Kirkland, ${ }^{6}$ and Stefan G. Tullius ${ }^{1}$ \\ 'Division of Transplant Surgery, Department of Surgery, Brigham and Women's Hospital, Harvard Medical School, Boston, Massachusetts, USA. Departments of Biochemistry and Physiology, Yong Loo Lin \\ School of Medicine, National University of Singapore, Singapore. ${ }^{3}$ Singapore Institute for Clinical Sciences, Singapore. ${ }^{4}$ Agency for Science, Technology and Research (A*STAR), Singapore. ${ }^{5}$ Buck Institute for \\ Research on Aging, Novato, California, USA. ${ }^{6}$ Robert and Arlene Kogod Center on Aging, Mayo Clinic, Rochester, Minnesota, USA.
}

\begin{abstract}
Donor age and recipient age are factors that influence transplantation outcomes. Aside from age-associated differences in intrinsic graft function and alloimmune responses, the ability of young and old cells to exert either rejuvenating or aging effects extrinsically may also apply to the transplantation of hematopoietic stem cells or solid organ transplants. While the potential for rejuvenation mediated by the transfer of youthful cells is currently being explored for therapeutic applications, aspects that relate to accelerating aging are no less clinically significant. Those effects may be particularly relevant in transplantation with an age discrepancy between donor and recipient. Here, we review recent advances in understanding the mechanisms by which young and old cells modify their environments to promote rejuvenation- or aging-associated phenotypes. We discuss their relevance to clinical transplantation and highlight potential opportunities for therapeutic intervention.
\end{abstract}

\section{Introduction}

Cellular aging is a complex process controlled by a combination of cell-intrinsic and environmental conditions. Young and old cells appear to be capable of regulating aging in a nonautonomous manner, with young cells having the capacity to promote tissue regeneration and old cells inducing aging-associated cellular and tissue dysfunction. These effects are not limited to the immediate microenvironment, as experimental models demonstrate that young and old cells exert both local and systemic influences when placed into age-asynchronous environments.

Initial insights into the physiological consequences of combining cells of different ages were predominantly investigated in experimental heterochronic parabiosis models, in which the circulatory systems of animals of different ages are joined. Interestingly, reciprocal effects occur, with both young and old parabiont partner animals being affected by the circulatory connection. While the regenerative capacity of muscle tissue in the heterochronic old animal improved following connection to a young partner animal, reciprocal effects were observed in young parabionts, who exhibited compromised rates of muscle progenitor divisions subsequent to joining with old partner animals $(1,2)$. Other tissue types may also be subject to such changes, as neurogenesis (3), hepatic regeneration, bile acid production (4), vascular calcification and remodeling $(5,6)$, and hematopoietic system structure (7) have all been shown to be altered in heterochronic models.

Several hypotheses as to how young and old cells may alter their environments to promote these effects have been proposed. In particular, studies have attempted to dissect the relative con-

Conflict of interest: A patent application, Methods and Materials for Improving Transplant Outcomes (US Provisional Patent 62/694,849), has been filed (JLK and SGT).

Reference information: J Clin Invest. 2019;129(1):4-11.

https://doi.org/10.1172/JCl123946. tributions of secreted factors versus cells in contributing to these phenomena. Cell signaling pathways that have been implicated in the regulation of age-related phenotypes may be modulated by soluble factors that interact with pathway receptors. Factors alone, however, may not be the sole mechanism by which aging-related phenotypes are transferred. Notably, adult stem cells transplanted into recipients are capable of differentiating into multiple tissue types and may contribute to recipient organ and tissue function (8). However, it is unclear whether these cells impact recipient organ function predominantly by acting as a local source of secreted soluble factors, or in fact by integrating into and contributing to target organ function.

Heterochronic parabiosis studies (and subsequently more refined studies) have opened a new vein of aging research, and one with therapeutic potential. An obvious potential therapeutic direction involves direct transfusion of blood or plasma alone, which may be sufficient to transfer factors that confer rejuvenation or aging $(9,10)$. An alternative approach involves either identifying rejuvenation factors in young blood and supplying them directly, or identifying aging factors and selectively removing them. Application of therapeutics that target senescent cells may also be beneficial, as these cells may be involved in the production and secretion of many of these aging and often inflammatory factors.

In science, the new often causes rethinking of the old. While longevity therapeutics have been developed based on the findings of heterochronic parabiosis and related studies, it is also useful to think about how these new insights into aging relate to a large collective of observations from research in transplant biology, a long and well-established therapeutic field that has positively impacted individuals with a wide range of diseases. It has been repeatedly demonstrated that the age of the transplant donor affects outcome, with grafts from older individuals showing inferior outcomes thought to be mainly related to an inferior quality. 


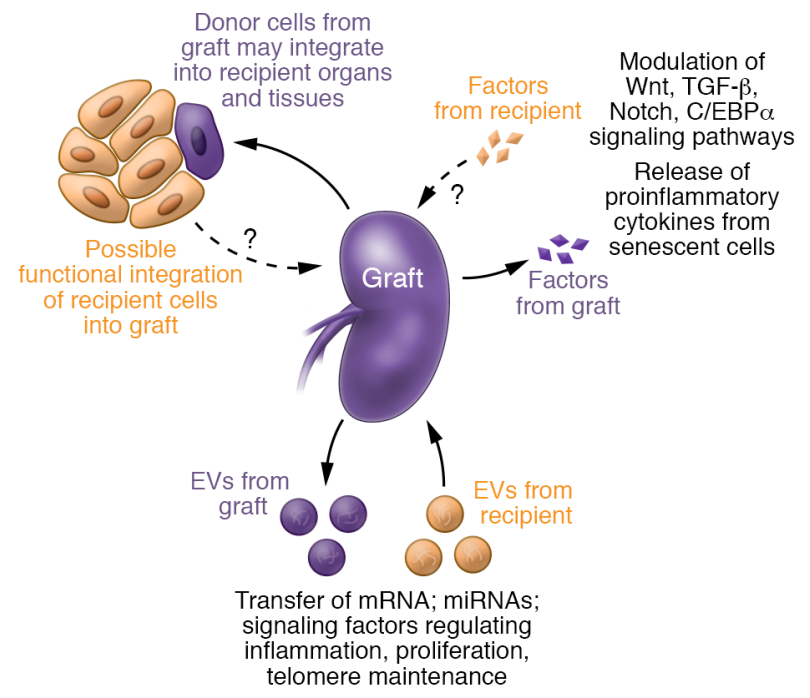

To date, transplantation studies have largely focused on how the recipient's environment modulates the graft (11). However, studies thus far have largely overlooked the graft's effects on the recipient environment. At least in theory, in settings where young and old tissues cohabitate, donor cells and recipient environment may have bidirectional effects leading to either rejuvenation or accelerated aging. Here, we juxtapose aging parabiosis studies with transplant research and speculate about the extent to which pathways identified from parabiosis studies influence organ, tissue, and cell transplantation. We suggest studies to refine the efficacy of transplant biology when old or young organs are used and discuss specific mechanisms and pathways of relevance. There is a strong possibility that insights from aging research may inform and accelerate our understanding of transplant biology. Similarly, insights from a wealth of transplant studies may modify thinking in the field of aging biology.

\section{Mechanisms underlying transfer of aging or rejuvenation}

Factor-mediated contributions. Candidate mediators of aging properties include soluble factors, cells, and cellular components (Figure 1). A key role for circulating soluble factors or cellular components is strongly supported by the demonstration of neurological rejuvenation in old mice following the administration of blood or plasma alone $(9,10)$. The identities of rejuvenating factors and factors that induce age-related changes remain a matter of debate. It is also unknown whether the same factors affect different tissues identically.

Multiple candidate blood-borne factors have been identified as mediators of rejuvenating or accelerated aging with phenotypic changes assessed by various proteomic approaches, including antibody arrays and mass spectrometry-based technologies $(3,10$, 12-14). In addition to soluble signaling factors affecting Wnt and TGF- $\beta$ signaling cascades, which have previously been implicated in the regulation of aging, a wide range of additional components, including matrix metalloproteases, cytokines, growth factors, and extracellular vesicles, appear to be important constituents of secretory profiles. Indeed, transcriptional profiling indicates that secreted factors constitute a large proportion of genes upregulat-
Figure 1. Mechanisms of transferring rejuvenation and aging. Several mediators of rejuvenation and aging may be transferred between young and old individuals. Donor cells may not only be capable of secreting factors that affect surrounding cells, but may also integrate into and contribute to tissue and organ function. Cells from the transplant recipient may also integrate into grafted tissue. Extracellular vesicles containing nucleic acids (predominantly mRNAs and miRNAs), proteins, and lipid products are capable of fusing with target cells to influence cellular behavior. Additionally, soluble factors secreted from cells can modulate signaling pathways implicated in the regulation of aging.

ed in response to a diverse array of senescence-promoting stimuli (reviewed in ref. 15).

Experimental manipulation of Wnt signaling has been shown to affect aging-associated phenotypes $(2,16)$, with enhanced signaling accelerating development of aging phenotypes. Recently, a definitive role for Wnt regulators in mediating the transmission of age-associated phenotypes has also been demonstrated with the identification of complement $\mathrm{C} 1 \mathrm{q}$ as a candidate age-promoting systemic factor. Complement C1q, a systemic factor that accumulates in multiple tissues with aging, has been shown to act as an agonist of canonical Wnt signaling by binding Frizzled receptors. Notably, increased circulating C1q levels were associated with decreased skeletal muscle regeneration and increased fibrosis in animal models (12).

Modulators of TGF- $\beta$ signaling may mediate additional effects. Growth differentiation factor 11 (GDF11), an activator of TGF- $\beta$ signaling, has been identified as another candidate promoting rejuvenation. Initially identified in a proteomic screen of factors that were more prevalent in sera of young mice relative to those of old mice (13), GDF11 was shown to reduce cardiac hypertrophy and age-associated defects in muscle regeneration $(13,17)$. Moreover, GDF11 promoted neurogenesis and vascular remodeling (6), with effects apparently mediated via the TGF- $\beta$ pathway. However, subsequent studies indicated that GDF11 levels increase with age, promoting aging-related phenotypes (18). Those findings contradicted the results of earlier studies, which have since been attributed to off-target effects on myostatin (GDF8), another member of the TGF- $\beta$ signaling pathway that is closely related to GDF11 (19). Activin A, which is also closely related to myostatin and GDF11, can be secreted by senescent cells and can bind to the same receptor (20). Activin A increases in adipose tissue with aging, causing stem and progenitor cell dysfunction and contributing to age-related insulin resistance. Further work remains to determine the precise role for GDF11, activin A, or myostatin in mediating cellular rejuvenating effects. Nevertheless, these results support the involvement of circulating factors that modulate TGF- $\beta$ signaling to affect aging or rejuvenation.

Several other signaling pathways, including Notch and $\mathrm{C} / \mathrm{EBP} \alpha$, have also been implicated in the regulation of rejuvenation and aging in heterochronic parabiosis experiments, though 
the individual candidate circulating factors modulating these pathways remain unidentified. While the inhibition of Notch signaling promoted skeletal muscle regeneration, $\mathrm{C} / \mathrm{EBP} \alpha$ inhibition promoted hepatic regeneration (1).

One characteristic of senescent cells is the secretion of a large number of factors. Regardless of the mechanism that induces senescence, proinflammatory cytokines (21), particularly IL-6 and IL-8, are components of the senescence-associated secretory phenotype (SASP) (22), supporting the involvement of immune response pathways in the cellular response to SASP exposure. In studies of circulating factors that promote the development of aging phenotypes, both CCL11, a plasma chemokine for neutrophils, and $\beta_{2}$-microglobulin, a component of the MHC class I complex, have been implicated as candidates for inhibition of neurogenesis and cognitive function in old mice $(3,14)$.

Coculture experiments of senescent cells demonstrate that the consequences of SASP exposure may depend, at least in part, on the duration of exposure to SASP factors (23). Acute exposure of neighboring cells to SASP factors appears to provoke cellular regeneration and repair responses, whereas chronic exposure may induce features of senescence that promote inflammation or tumorigenesis, indicating that the consequences of SASP factors are context-dependent, rather than promoting either rejuvenating or aging. As these data suggest that suppression of inflammation supports the prevention of aging, it is interesting to note that a beneficial role for immune responses also exists in alleviating aging phenotypes. Therefore, the consequences of altering these pathways are likely to be complex, with beneficial and detrimental consequences both associated with engagement of pathways that are context-dependent.

Cell-mediated contributions. Although soluble factors may play a dominant role in mediating rejuvenating and aging phenotypes, cellular contributions cannot be excluded. Results of lineage tracing experiments following transplanted tissues indicate the potential for transferred adult stem cells to incorporate into recipient organs. Following hematopoietic stem cell (HSC) transplants, donor cells have been identified in multiple organs, including muscle $(24)$, liver $(25,26)$, myocardium $(27,28)$, and endothelium (29). As HSCs can be induced to transdifferentiate in vitro into multiple tissue types (30), these findings have been interpreted as evidence that transplanted adult stem cells may integrate into and contribute functionally to recipient organ function. Given these observations, it is likely that recipient cells also incorporate into the graft and may have functional consequences; however, this has not been well characterized thus far and remains to be determined.

Nevertheless, integration into adult tissues remains difficult to prove, particularly in a clinical setting. Some studies have demonstrated donor HSC integration into liver and skin, using the $\mathrm{Y}$ chromosome as a marker for donor cell tracing in sexmismatched transplants from male donors to female recipients (31). As the frequency of HSC integration appears to be relatively low, transdifferentiation may be a relatively rare occurrence (32, 33). Furthermore, the persistence of these cells may be limited, as recipient-derived cells were more frequently identified in early compared with later transplant biopsies (34). Notably, in models of stem cell transplantation for myocardial disease, the majority of donor cells underwent apoptosis within several days (35).
These observations favor the local production of factors by transplanted stem cells in promoting either rejuvenation or aging. Following homing of donor cells in response to chemokines produced by damaged tissues, the localized release of such factors may result in augmented consequences compared with a systemic administration. Additional benefits may also derive from direct cell-cell signaling mechanisms or, alternatively, stem cell differentiation and contribution to recipient tissues.

Few experiments have examined the fate of senescent cells in transplants from older donors to younger recipients to determine whether donor-derived cells migrate to distant tissues. One may speculate that the effects of senescent cells on surrounding tissues and the systemic environment appear to be predominantly mediated by the release of factors, though direct cell-cell signaling may increase local influences (21).

Additional mechanisms. Aside from factors and cells, further means of communication may occur through extracellular vesicles (EVs). These cellular byproducts, containing mRNAs, miRNAs, proteins, and lipid components, contribute to cell-cell communication in both a local and a distant fashion, with functional consequences (reviewed in refs. 36, 37). While EVs are secreted from numerous cell types, including stem cells and senescent cells, the number of EVs secreted increases dramatically with senescence. The content of EVs also depends on source cells, as various factors, including galectin-3, and certain miRNAs that promote stem cell function are present in higher amounts in EVs from young cells than in those from senescent cells (38). EVs from senescent cells have been proposed to be capable of promoting several agingassociated processes, including increased mitogenic signaling, proliferation, inflammatory gene expression, and telomere dysregulation in neighboring cells. Accordingly, in vitro experiments demonstrated that EV depletion from tumor cell serum reduced its protumorigenic effects (39).

\section{Clinical implications}

The ability of youthful and senescent cells to regulate the aging of surrounding cells and tissues has potential significance for solid organ and bone marrow transplantation, clinical scenarios in which donors and recipients are not necessarily of similar age. In age-mismatched transplants, both the allograft and the recipient environment have the capacity to influence each other, suggesting a possible two-way communication with implications not only for graft survival and overall recipient outcome, but potentially also for systemic aging or rejuvenation of the recipient.

Effects of donor age. Few studies have examined the association of donor age with aging-associated phenotypes, aside from those related to graft function and overall survival. In HSC transplantation, older HSCs have compromised replicative capacity, with accelerated telomere shortening, increased ROS, and DNA damage accumulation, likely exacerbated by the replicative stress incurred by the transplantation process (40-42). Old HSCs also exhibit reduced rates of homing and engraftment with impaired differentiation capacities toward the lymphoid lineage (43). Clinically, those effects may lead to higher rates of graft-versus-host disease and disease relapse, which impact recipient survival and are associated with older donor age, as observed in both conventional and reduced-intensity protocols for HSC transplantation (44-47). 
In addition to these intrinsic alterations in donor HSC function that result from advanced donor age, there may be additional sequelae of older donor age affecting physiological processes in the recipient. Studies in animal models demonstrated that recipients of young-donor bone marrow had higher levels of bone mineral density and markers of new bone formation than those who received old-donor bone marrow. Overall lifespan was also increased in comparison with control animals or recipients of old-donor bone marrow (48). Although molecular mechanisms involved in mediating these effects on lifespan have not been examined specifically, prior studies have established a role for mesenchymal stem cells in promoting rejuvenating effects, including hippocampal neurogenesis, alleviation of autoimmune disease, and improved myocardial function $(49,50)$. These cells appear to contribute to rejuvenation predominantly by secreting chemokines, but may also contribute via cell fusion and differentiation into various lineages.

Although the ability of solid organ allografts to promote aging or rejuvenation-associated phenotypes in recipients has not been specifically examined, experimental models support the capacity of transplanted cells to induce aging-associated features in recipients. Injection of senescent cells in the knees of young mice causes premature development of osteoarthritis-like symptoms (51). Notably, transplanting senescent (versus nonsenescent) adipose mesenchymal cells into the peritoneal cavity of young mice, such that only 1 out of 10,000 cells in the recipient animal is a transplanted senescent cell, is sufficient to cause muscle weakness and other frailty-like disabilities that persist for at least 6 months (52). Furthermore, middle-aged mice transplanted with senescent cells have a decreased lifespan compared with age-matched mice transplanted with nonsenescent cells, with comparable causes of death in both groups, suggesting an acceleration of age-related pathologies rather than the promotion of any single disease. While labeled transplanted senescent cells remained within the abdominal cavity, senescent cells arising from the recipients' own cells developed in distant sites. Those observations demonstrate that spread of senescence can occur from localized populations of transplanted senescent cells, with implications for transplanted organs that carry senescent cells with them.

It is somewhat difficult to determine whether these observations in experimental models have relevance in the clinical setting for solid organ transplantation, as clinical studies comparing outcomes of transplants from young donors and old donors have thus far largely focused on graft survival and associated characteristics. Some studies suggest that outcomes of kidney transplants from young donors and old donors are largely comparable, provided that they are corrected for intrinsic differences in graft function as assessed by pretransplant histological analysis of the donor kidney, and that allocation methods consider matching donor and recipient age to some extent $(53,54)$. However, the outcomes assessed in these analyses aside from overall survival and graft survival are creatinine clearance and urine protein excretion, characteristics that predominantly reflect function of the graft rather than overall recipient physiological status. Analyses of lung, heart, and liver transplant outcomes based on donor age have also similarly focused largely on graft function.

Effects of recipient age. The effect of recipient age on graft function following transplantation has been noted in multiple cell and tissue types, with youthful or aged environments determining the regeneration capacity of transplanted tissue in animal models (11, $55)$. Notably, the regenerative capacity of skeletal muscle grafts in mouse models was found to correspond to the age of recipients rather than the age of the donor. Muscle grafts derived from old donors regained the capacity to regenerate when transplanted into young recipients; however, when grafts from young donors were transplanted into aged recipients, regenerative capacity appeared compromised (11). Regenerative properties of mammary gland tissue transplants were also found to depend on recipient age, as both young- and old-donor muscle grafts exhibited similar growth rates when transplanted into young recipients with minimal growth when placed into old recipients, though contributions of hormonal differences and replicative senescence to these results could not be excluded in this study (55).

Older recipient age also appears to increase disease recurrence subsequent to HSC transplantation in leukemia models, likely as a consequence of altered signaling in the HSC niche that occurs with aging $(56,57)$. Stromal cells in the niche have been shown to undergo changes in secretory profile with aging, including decreases in osteopontin secretion (58), that inhibit HSC function. Integrity of the vasculature that feeds the niche is also likely critical for maintenance of HSC function, as Notch-dependent vessel formation appears to be critical for HSC function (59), and aged HSCs can be restored by endothelial transplantation (60). Additional features of the niche microenvironment, including sympathetic nerve fiber function (61), are likely also important for supporting HSC function and decline with age.

Senescence can also be induced in recipients during the requisite preconditioning process, and may not solely be a result of aging. Chemotherapy and radiotherapy, which are often used during the preconditioning process for HSC transplant recipients, induce senescence in HSCs and cause long-term inhibition of HSC self-renewal and HSC reserves, thus adding additional considerations to the assessment of effects of recipient age (62).

In other models, it has been noted that face allografts exhibit structural changes that resemble accelerated aging, although these changes may very well be related to surgical aspects of transplantation and immunosuppression (63). Similarly, the accelerated senescence observed in adult organs transferred to pediatric recipients may also reflect consequences of immunosuppression and replicative stress (64). Despite the frequency of differing donor and recipient ages in solid organ transplantation, there has been little research on the effects of recipient age on donor organ function.

\section{Opportunities for intervention}

The potential of young or old cells to promote rejuvenation or inhibit senescence indicates that there may be interventional approaches that recapitulate these age-associated influences. Indeed, clinical trials are currently under way assessing the consequences of transfusing young serum, and of stem cell therapies (reviewed in ref. 65). Although early results examining effects of infusing stem cells indicate that such therapies may reduce levels of aging-associated inflammatory biomarkers and increase select measures of physical performance (66), additional studies are required to determine their specific and long-term consequences. 


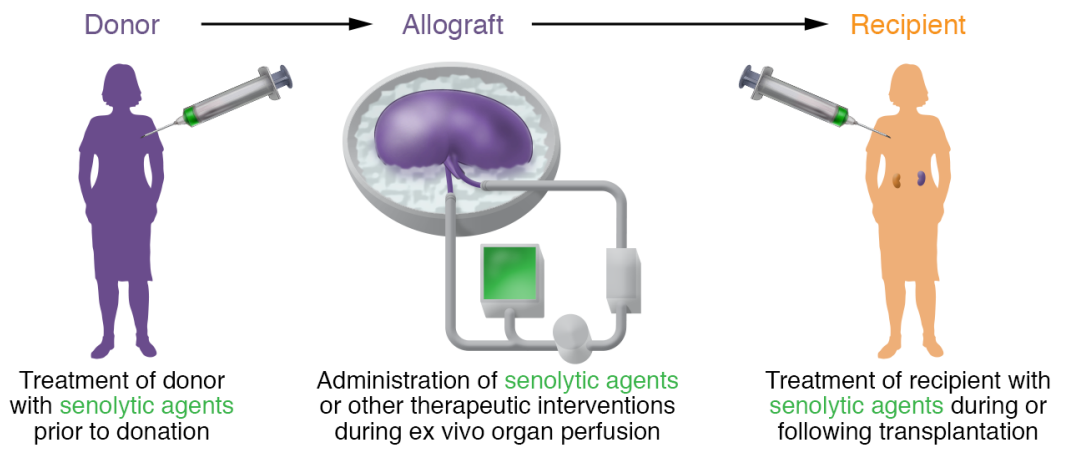

Figure 2. Potential opportunities for therapeutic intervention. Left: Older transplant donors may be treated with senolytic agents before organ donation to a younger recipient. Senolytics with a minimal side effect profile are desirable for such applications. Middle: Treatment of the allograft with senolytic agents following organ procurement and prior to transplantation provides additional opportunities to intervene for the administration of senolytics, potentially via the addition of such compounds to organ preservation solutions and perfusates. Recent development of novel organ preservation methods permitting an extended duration of ex vivo organ maintenance may support not only senolytic treatment, but also additional means of therapeutic intervention. Right: Transplant recipients can also be treated with senolytics or senomorphics at the time of or subsequently to transplantation. Initial studies of these compounds indicate that the extent of their direct effects is specific to senescent cells.

Experimental studies support the concept that selective depletion of senescent cells can be beneficial. At the cellular level, senescent cell depletion leads to rejuvenation of stem cells, promoting proliferation in both HSC and hair stem cell models (67, 68). These changes also have physiological consequences. Using a genetic p16(INK-ATTAC) model targeting 16 ${ }^{\text {Ink4a }}$-positive cells (including many senescent cells) for apoptosis, age-associated conditions including frailty, metabolic dysfunction, age-related osteoporosis, or pulmonary fibrosis can be delayed or alleviated $(52,69-74)$. Predominant interventional strategies include a selective induction of apoptosis of senescent cells by administration of senolytic agents (75-81), in addition to targeting of the SASP machinery in senescent cells with senomorphic drugs (20, 78-80). Moreover, senolytic drugs have shown efficacy in alleviating frailty in progeroid and naturally aging mice; increasing the remaining lifespan while alleviating radiation-induced dysfunction; alleviating tau-induced neurocognitive disease, bleomycin-induced pulmonary fibrosis, hepatic steatosis, liver dysfunction and fibrosis, age- and high-fat diet-induced vascular hyporeactivity and calcification; arresting and partially reversing age-related osteoporosis; and enhancing cardiac ejection fraction in old mice $(52,72-75,78-$ $80,82-84)$. Since it can take up to 6 weeks for new senescent cells to form, senescent cell-depleting therapies require infrequent administration relative to secretome-targeting compounds that must be given repeatedly $(78,79)$. Several senolytic compounds are currently being investigated for their clinical application. As promising as senolytics appear to be in preclinical models, some senolytic agents, such as navitoclax, can have serious off-target effects, although others have fewer known adverse effects. Most (or perhaps all) senolytics exhibit differing specificities depending on the cell type from which the senescent cells originated. Thus, for a clinical application, chemical optimization, testing of which senolytic agent to use for which indication, testing of clinical side effects and safety in humans, and optimization of dosing regimens need to be performed. In the future, it may also be possible for senomorphic and senolytic drugs to selectively promote the beneficial aspects of the SASP in enhancing tissue repair, while still mitigating negative consequences, such as promoting inflammation and cancer (85).

Aside from interventions that directly target senescent cells, other compounds with alternative mechanisms appear to also have senomorphic properties. Inhibitors of the mammalian target of rapamycin (mTOR) pathway extend both lifespan and healthspan in a variety of animal models (86). Rapamycin, the canonical mTOR inhibitor, has been shown to reduce inflammation with aging, preserve adult stem cell function, and enhance cellular stress resistance. Induction of senescence appears to involve activation of the TOR pathway, though the mechanism by which mTOR inhibition slows aging processes remains a matter of debate.

In addition to these strategies, an improved understanding of the molecular pathways that regulate aging and rejuvenating phenotypes may lead to other therapeutic opportunities. This aspect of aging research remains in its infancy, and the utility of rejuvenating strategies needs to be determined.

Applications in transplantation. The recognition that donor age and recipient age affect organ transplantation outcomes has led to the initiation of programs attempting to allocate organs in a manner that matches donor age and recipient age. This rationale is predominantly based on superior functions of younger compared with older organs. In addition to the effects on alloimmune responses, age-matched transplants may also reduce some of the potential adverse consequences of introducing senescent cells when transplanting old-donor organs into young recipients.

Rapamycin is used clinically in transplantation to prevent rejection, and it is interesting to speculate whether individuals exposed to long-term administration of rapamycin may have altered aging properties. While the immunosuppressive effects of rapamycin prevent graft rejection, they also decrease senescent cell accumulation that occurs as a result of immune surveillance. However, age-related effects of rapamycin may be confounded by compromised health status of transplant recipients and the presence of other immunosuppressive drugs. Known adverse effects of rapamycin also include myelosuppression (87), which can be significant particularly in the context of HSC transplantation.

Additional benefits may be obtained from treating donors, recipients, or allografts with therapies that promote rejuvenation or target senescent cells. Transplanting senescent cells into young mice may shorten survival while inducing age-related phenotypes and pathologies $(51,52)$. These effects may also be exacerbated by vascular shear stress, induced upon the connection of donor organ vessels to recipient vasculature. As shown in an animal model of arteriovenous fistulas, shear stress promotes premature senescence in the vein after fistula creation (88). Therefore, organ or cell transplantation from old donors harboring senescent cells may induce age-associated dysfunctions in younger recipients. However, administering senolytics at the time of transplanting senescent 
mesenchymal cells into the abdominal cavity of mice prevented frailty and improved survival. Even after frailty has already developed, senolytics are still effective, suggesting that it is possible to overcome some of the problems that may accelerate aging-related processes when organs are transplanted from old donors.

For solid organ transplantation, several windows of therapeutic targeting exist, including treatment of the donor, treatment of the recipient, and treatment of the graft itself (Figure 2). Treatment of the donor with senolytic agents prior to donation, for example, is one potential means of depleting senescent cells within the allograft. This concept would likely have more relevance in the setting of living-donor transplantation, as deceased-donor transplantation situations do not permit the advanced planning required for administration of such compounds. At this time, adverse effects of certain senolytic agents, such as cytopenias caused by navitoclax or, possibly, delayed wound healing (77), limit the application of this strategy, as minimum harm to the donor is required.

Transplant recipients may also receive senolytic or senomorphic agents following transplantation, though targeted effects specifically on the graft cannot be achieved with current methods.

Treatment of the allograft itself, following organ procurement and prior to transplantation into the recipient, may provide another opportunity for intervention. Although current protocols focus on minimizing the duration of cold ischemia to reduce its adverse effects on allograft function, brief treatments with senolytic agents (potentially by adding them to organ preservation solutions) are likely feasible and provide a unique opportunity for targeted delivery of these compounds. In addition, emerging preservation concepts are allowing for prolonged ischemic times, permitting the administration of such agents.

Indeed, the emerging technology of ex vivo organ perfusion, currently under development as a means of increasing organ usage in transplantation, may provide additional flexibility. Such systems will not only allow assessment of organ function prior to transplantation, but also provide a platform for therapeutic administration (89). In support of this concept, a brief exposure of freshly procured clinical adipose tissue to senolytics has been shown to be sufficient to reduce senescent cell abundance by apoptosis, while leaving nonsenescent cells intact (52). In addition to small molecules, nucleic acid- and viral vector-based therapeutics, immunization, and cellular therapies are being developed, potentially allowing for more specific targeting of senescent cells.

\section{Conclusions}

The ability of youthful and senescent cells to exert systemic rejuvenating or advanced aging has significant clinical implications. The identification of circulating factors and cells as potential mechanisms by which these cells exert their effects has been instrumental in developing potential therapeutic interventions that promote rejuvenation while minimizing effects of aging. Plasma and blood transfusions promoting rejuvenation, as well as senolytic and senomorphic drugs, are currently under investigation for clinical use. Further work that identifies additional mediators of rejuvenation and aging will likely not only clarify how these processes are regulated, but also highlight new opportunities for intervention. As the elderly are increasingly becoming transplant donors and recipients, these insights should help clarify the appropriate applications for therapeutics that promote rejuvenation and target senescent cells.

\section{Acknowledgments}

SGT was supported by NIH grant RO1AG039449 and a generous unrestricted grant by Kenneth and Melissa Crane. BKK was supported by NIH grant AG050441. JLK was supported by the Connor Group and Robert J. and Theresa W. Ryan, NIH grants AG13925 and AG49182, a Glenn/American Federation for Aging Research (AFAR) BIG Award, and the Ted Nash Long Life and Noaber Foundations.

Address correspondence to: Stefan G. Tullius, Division of Transplant Surgery and Transplant Surgery Research Laboratory, Brigham and Women's Hospital, Harvard Medical School, 75 Francis Street, Boston, Massachusetts 02115, USA. Phone: 617.732.6446; Email: stullius@bwh.harvard.edu.
1. Conboy IM, Conboy MJ, Wagers AJ, Girma ER, Weissman IL, Rando TA. Rejuvenation of aged progenitor cells by exposure to a young systemic environment. Nature. 2005;433(7027):760-764.

2. Brack AS, et al. Increased Wnt signaling during aging alters muscle stem cell fate and increases fibrosis. Science. 2007;317(5839):807-810.

3. Villeda SA, et al. The ageing systemic milieu negatively regulates neurogenesis and cognitive function. Nature. 2011;477(7362):90-94.

4. Uchida K, Takase H, Nomura Y, Satoh T, Igimi H, Takeuchi N. Bile acid metabolism in young-old parabiotic rats. Lipids. 1997;32(4):383-390.

5. Adams KH, Blish RC, Vreeland T. Second-order pyramidal slip in zinc single crystals. Mater Sci Eng. 1967;2(4):201-207.

6. Katsimpardi L, et al. Vascular and neurogenic rejuvenation of the aging mouse brain by young systemic factors. Science. 2014;344(6184):630-634.

7. Sidorenko AV, Gubrii IB, Andrianova LF, Macsijuk TV, Butenko GM. Functional rearrangement of lymphohemopoietic system in heter- ochronically parabiosed mice. Mech Ageing Dev. 1986;36(1):41-56

8. Kørbling M, Estrov Z. Adult stem cells for tissue repair - a new therapeutic concept? $N$ Engl JMed. 2003;349(6):570-582.

9. Villeda SA, et al. Young blood reverses age-related impairments in cognitive function and synaptic plasticity in mice. Nat Med. 2014;20(6):659-663.

10. Castellano JM, et al. Human umbilical cord plasma proteins revitalize hippocampal function in aged mice. Nature. 2017;544(7651):488-492.

11. Carlson BM, Faulkner JA. Muscle transplantation between young and old rats: age of host determines recovery. Am J Physiol. 1989; 256(6 pt 1):C1262-C1266.

12. Naito AT, et al. Complement C1q activates canonical Wnt signaling and promotes aging-related phenotypes. Cell. 2012;149(6):1298-1313.

13. Loffredo FS, et al. Growth differentiation factor 11 is a circulating factor that reverses age-related cardiac hypertrophy. Cell. 2013;153(4):828-839.

14. Smith LK, et al. $\beta 2$-Microglobulin is a systemic pro-aging factor that impairs cognitive function and neurogenesis. Nat Med. 2015;21(8):932-937.

15. Krtolica A, Campisi J. Cancer and aging: a model for the cancer promoting effects of the aging stroma. Int J Biochem Cell Biol. 2002;34(11):1401-1414.

16. Liu H, et al. Augmented Wnt signaling in a mammalian model of accelerated aging. Science. 2007;317(5839):803-806.

17. Sinha M, et al. Restoring systemic GDF11 levels reverses age-related dysfunction in mouse skeletal muscle. Science. 2014;344(6184):649-652.

18. Egerman MA, et al. GDF11 increases with age and inhibits skeletal muscle regeneration. Cell Metab. 2015;22(1):164-174.

19. Schafer MJ, et al. Quantification of GDF11 and myostatin in human aging and cardiovascular disease. Cell Metab. 2016;23(6):1207-1215.

20. Xu M, et al. JAK inhibition alleviates the cellular senescence-associated secretory phenotype and frailty in old age. Proc Natl Acad SciU S A. 2015;112(46):E6301-E6310.

21. Coppé JP, et al. Senescence-associated secretory 
phenotypes reveal cell-nonautonomous functions of oncogenic RAS and the p53 tumor suppressor. PLoS Biol. 2008;6(12):2853-2868.

22. Malaquin N, Martinez A, Rodier F. Keeping the senescence secretome under control: molecular reins on the senescence-associated secretory phenotype. Exp Gerontol. 2016;82:39-49.

23. Ritschka B, et al. The senescence-associated secretory phenotype induces cellular plasticity and tissue regeneration. Genes Dev. 2017;31(2):172-183.

24. Gussoni E, et al. Dystrophin expression in the $\mathrm{mdx}$ mouse restored by stem cell transplantation. Nature. 1999;401(6751):390-394.

25. Lagasse E, et al. Purified hematopoietic stem cells can differentiate into hepatocytes in vivo. Nat Med.2000;6(11):1229-1234.

26. Wang X, Ge S, McNamara G, Hao QL, Crooks GM, Nolta JA. Albumin-expressing hepatocyte-like cells develop in the livers of immune-deficient mice that received transplants of highly purified human hematopoietic stem cells. Blood. 2003;101(10):4201-4208.

27. Orlic $\mathrm{D}$, et al. Bone marrow cells regenerate infarcted myocardium. Nature. 2001;410(6829):701-705.

28. Jackson KA, et al. Regeneration of ischemic cardiac muscle and vascular endothelium by adult stem cells. J Clin Invest. 2001;107(11):1395-1402.

29. Grant MB, et al. Adult hematopoietic stem cells provide functional hemangioblast activity during retinal neovascularization. Nat Med. 2002;8(6):607-612.

30. Krause DS, et al. Multi-organ, multi-lineage engraftment by a single bone marrow-derived stem cell. Cell. 2001;105(3):369-377.

31. Körbling M, et al. Hepatocytes and epithelial cells of donor origin in recipients of peripheral-blood stem cells. N EnglJ Med. 2002;346(10):738-746.

32. Fogt F, Beyser KH, Poremba C, Zimmerman RL, Khettry U, Ruschoff J. Recipient-derived hepatocytes in liver transplants: a rare event in sex-mismatched transplants. Hepatology. 2002;36(1):173-176.

33. Schwerfeld-Bohr J, Chi H, Worm K, Dahmen U. Influence of hematopoietic stem cell-derived hepatocytes on liver regeneration after sexmismatched liver transplantation in humans. J Invest Surg. 2012;25(4):220-226.

34. Idilman $\mathrm{R}$, et al. Recipient-derived hepatocytes in sex-mismatched liver allografts after liver transplantation: early versus late transplant biopsies. Transplantation. 2004;78(11):1647-1652.

35. Toma C, Pittenger MF, Cahill KS, Byrne BJ, Kessler PD. Human mesenchymal stem cells differentiate to a cardiomyocyte phenotype in the adult murine heart. Circulation. 2002;105(1):93-98.

36. Takasugi M. Emerging roles of extracellular vesicles in cellular senescence and aging. Aging Cell. 2018;17(2):e12734.

37. Vader P, Breakefield XO, Wood MJ. Extracellular vesicles: emerging targets for cancer therapy. Trends Mol Med. 2014;20(7):385-393.

38. Weilner S, et al. Vesicular Galectin-3 levels decrease with donor age and contribute to the reduced osteo-inductive potential of human plasma derived extracellular vesicles. Aging (Albany NY). 2016;8(1):16-33.
39. Takasugi M, Okada R, Takahashi A, Virya Chen D, Watanabe S, Hara E. Small extracellular vesicles secreted from senescent cells promote cancer cell proliferation through EphA2. Nat Commun. 2017;8:15729.

40. Awaya $\mathrm{N}$, et al. Telomere shortening in hematopoietic stem cell transplantation: a potential mechanism for late graft failure? Biol Blood Marrow Transplant. 2002;8(11):597-600.

41. Yahata T, et al. Accumulation of oxidative DNA damage restricts the self-renewal capacity of human hematopoietic stem cells. Blood. 2011;118(11):2941-2950.

42. Cupit-Link MC, et al. Biology of premature ageing in survivors of cancer. ESMO Open. 2017;2(5):e000250.

43. Liang Y, Van Zant G, Szilvassy SJ. Effects of aging on the homing and engraftment of murine hematopoietic stem and progenitor cells. Blood. 2005;106(4):1479-1487.

44. Kollman C, et al. Donor characteristics as risk factors in recipients after transplantation of bone marrow from unrelated donors: the effect of donor age. Blood. 2001;98(7):2043-2051.

45. Flowers ME, et al. Comparative analysis of risk factors for acute graft-versus-host disease and for chronic graft-versus-host disease according to National Institutes of Health consensus criteria. Blood. 2011;117(11):3214-3219.

46. Nademanee A, et al. The outcome of matched unrelated donor bone marrow transplantation in patients with hematologic malignancies using molecular typing for donor selection and graftversus-host disease prophylaxis regimen of cyclosporine, methotrexate, and prednisone. Blood. 1995;86(3):1228-1234.

47. Mehta J, et al. Does younger donor age affect the outcome of reduced-intensity allogeneic hematopoietic stem cell transplantation for hematologic malignancies beneficially? Bone Marrow Transplant. 2006;38(2):95-100.

48. Shen J, Tsai YT, Dimarco NM, Long MA, Sun X, Tang L. Transplantation of mesenchymal stem cells from young donors delays aging in mice. $\mathrm{Sci}$ Rep. 2011;1:67.

49. Munoz JR, Stoutenger BR, Robinson AP, Spees JL, Prockop DJ. Human stem/progenitor cells from bone marrow promote neurogenesis of endogenous neural stem cells in the hippocampus of mice. Proc Natl Acad Sci US A. 2005;102(50):18171-18176.

50. Latham N, et al. Human blood and cardiac stem cells synergize to enhance cardiac repair when cotransplanted into ischemic myocardium. Circulation. 2013;128(11 suppl 1):S105-S112.

51. Xu M, et al. Transplanted senescent cells induce an osteoarthritis-like condition in mice. J Gerontol A Biol Sci Med Sci. 2017;72(6):780-785.

52. Xu M, et al. Senolytics improve physical function and increase lifespan in old age. Nat Med. 2018;24(8):1246-1256.

53. Remuzzi G, et al. Long-term outcome of renal transplantation from older donors. $\mathrm{N}$ Engl J Med. 2006;354(4):343-352.

54. Giessing M, et al. Outcomes of transplanting deceased-donor kidneys between elderly donors and recipients. J Am Soc Nephrol. 2009;20(1):37-40.
55. Young LJ, Medina D, DeOme KB, Daniel CW. The influence of host and tissue age on life span and growth rate of serially transplanted mouse mammary gland. Exp Gerontol. 1971;6(1):49-56.

56. Vas V, Senger K, Dörr K, Niebel A, Geiger $\mathrm{H}$. Aging of the microenvironment influences clonality in hematopoiesis. PLoS One. 2012;7(8):e42080.

57. Vas V, Wandhoff C, Dörr K, Niebel A, Geiger H. Contribution of an aged microenvironment to aging-associated myeloproliferative disease. PLoS One. 2012;7(2):e31523.

58. Guidi N, et al. Osteopontin attenuates agingassociated phenotypes of hematopoietic stem cells. EMBO J. 2017;36(7):840-853.

59. Kusumbe AP, et al. Age-dependent modulation of vascular niches for haematopoietic stem cells Nature. 2016;532(7599):380-384.

60. Poulos MG, et al. Endothelial transplantation rejuvenates aged hematopoietic stem cell function. JClin Invest. 2017;127(11):4163-4178.

61. Maryanovich $\mathrm{M}$, et al. Adrenergic nerve degeneration in bone marrow drives aging of the hematopoietic stem cell niche. Nat Med. 2018;24(6):782-791.

62. Wang Y, Schulte BA, LaRue AC, Ogawa M, Zhou D. Total body irradiation selectively induces murine hematopoietic stem cell senescence. Blood. 2006;107(1):358-366.

63. Kueckelhaus M, et al. Transformation of face transplants: volumetric and morphologic graft changes resemble aging after facial allotransplantation. Am J Transplant. 2016;16(3):968-978.

64. Hodgson R, Christophi C. What determines ageing of the transplanted liver? HPB (Oxford). 2015;17(3):222-225.

65. Ranganath SH, Levy O, Inamdar MS, Karp JM. Harnessing the mesenchymal stem cell secretome for the treatment of cardiovascular disease. Cell Stem Cell. 2012;10(3):244-258.

66. Tompkins BA, et al. Allogeneic mesenchymal stem cells ameliorate aging frailty: a phase ii randomized, double-blind, placebo-controlled clinical trial.J Gerontol A Biol Sci Med Sci. 2017;72(11):1513-1522.

67. Chang J, et al. Clearance of senescent cells by ABT263 rejuvenates aged hematopoietic stem cells in mice. Nat Med. 2016;22(1):78-83.

68. Yosef R, et al. Directed elimination of senescent cells by inhibition of BCL-W and BCL-XL. Nat Commun. 2016;7:11190.

69. Baker DJ, et al. Clearance of p16Ink4a-positive senescent cells delays ageing-associated disorders. Nature. 2011;479(7372):232-236.

70. Baker DJ, et al. Naturally occurring p16(Ink4a)-positive cells shorten healthy lifespan. Nature. 2016;530(7589):184-189.

71. Xu M, et al. Targeting senescent cells enhances adipogenesis and metabolic function in old age. Elife. 2015;4:e12997.

72. Roos CM, et al. Chronic senolytic treatment alleviates established vasomotor dysfunction in aged or atherosclerotic mice. Aging Cell. 2016;15(5):973-977.

73. Schafer MJ, et al. Cellular senescence mediates fibrotic pulmonary disease. Nat Commun. 2017;8:14532.

74. Farr JN, et al. Targeting cellular senescence 
prevents age-related bone loss in mice. Nat Med. 2017;23(9):1072-1079.

75. Zhu Y, et al. The Achilles' heel of senescent cells: from transcriptome to senolytic drugs. Aging Cell. 2015;14(4):644-658.

76. Zhu Y, et al. Identification of a novel senolytic agent, navitoclax, targeting the $\mathrm{Bcl}-2$ family of anti-apoptotic factors. Aging Cell. 2016;15(3):428-435.

77. Zhu Y, et al. New agents that target senescent cells: the flavone, fisetin, and the BCL-XL inhibitors, A1331852 and A1155463. Aging (Albany NY). 2017;9(3):955-963.

78. Kirkland JL, Tchkonia T. Cellular senescence: a translational perspective. EBioMedicine. 2017;21:21-28.

79. Kirkland JL, Tchkonia T, Zhu Y, Niedernhofer LJ, Robbins PD. The clinical potential of senolytic drugs. J Am Geriatr Soc. 2017;65(10):2297-2301. 80. Fuhrmann-Stroissnigg H, et al. Identification of
HSP90 inhibitors as a novel class of senolytics. Nat Commun. 2017;8(1):422.

81. Kirkland JL, Tchkonia T. Clinical strategies and animal models for developing senolytic agents. Exp Gerontol. 2015;68:19-25.

82. Musi N, et al. Tau protein aggregation is associated with cellular senescence in the brain [published online ahead of print August 20, 2018]. Aging Cell. https://doi.org/10.1111/acel.12840.

83. Ogrodnik M, et al. Cellular senescence drives age-dependent hepatic steatosis. Nat Commun. 2017;8:15691

84. Moncsek A, et al. Targeting senescent cholangiocytes and activated fibroblasts with B-cell lymphoma-extra large inhibitors ameliorates fibrosis in multidrug resistance 2 gene knockout ( $\left.\mathrm{Mdr}^{-/}\right)$ mice. Hepatology. 2018;67(1):247-259.

85. Tchkonia T, Zhu Y, van Deursen J, Campisi J, Kirkland JL. Cellular senescence and the senescent secretory phenotype: therapeutic opportuni- ties. J Clin Invest. 2013;123(3):966-972.

86. Kennedy BK, Lamming DW. The mechanistic target of rapamycin: the grand conducTOR of metabolism and aging. Cell Metab. 2016;23(6):990-1003.

87. Webster AC, Lee VW, Chapman JR, Craig JC. Target of rapamycin inhibitors (sirolimus and everolimus) for primary immunosuppression of kidney transplant recipients: a systematic review and meta-analysis of randomized trials. Transplantation. 2006;81(9):1234-1248.

88. Nath KA, et al. The murine dialysis fistula model exhibits a senescence phenotype: pathobiologic mechanisms and therapeutic potential [published online ahead of print July 18, 2018]. Am JPhysiol Renal Physiol. https://doi.org/10.1152/ ajprenal.00308.2018.

89. Van Raemdonck D, Neyrinck A, Cypel M, Keshavjee S. Ex-vivo lung perfusion. Transpl Int. 2015;28(6):643-656. 